1982). ${ }^{7}$ Equally noteworthy was the increasingly large number of couples with few or no children who opted for sterilisation: three quarters had two children or fewer and more than one in eight had none at all.

Failing the discovery of the perfect contraceptive-or further serious adverse research findings-current trends in contraceptive usage are unlikely to change radically in the foreseeable future. Men and women will be forced to go through the fertile period of life continually balancing the risks of efficacy and safety. In some cases this will mean a switch to a method which reduces worrying side effects but increases the risk of pregnancy; or couples may opt for sterilisation, which offers greater peace of mind in relation to unwanted pregnancy, but at the expense of a reprieve if there is a change of heart.

Either way, the decision a couple makes should be based on a calculated risk acceptable to both partners. The principle of informed choice for the layman is perhaps more appropriate to birth control than to any other means of preventive health care; yet it is possible only with good information. The sort of panic measures induced by media treatment of studies reporting adverse effects of methods might be avoided in the future by more balanced and circumspect reporting of all the important research in the subject.

Kaye Wellings Research officer

ANgela Mills National medical officer

Family Planning Association, London WIN 7RJ

1 Cartwright A. Parents and family planning services. London: Routledge Kegan Paul, 1970. Pike MC, Henderson BE, Krailo MD, Duke A. Breast cancer in young women and the use of oral contraceptives: possible modifying effect of formulation and age at use. Lancet 1983;ii:926-30. essey MP, Lawless M, McPherson K, Yeates D. Neoplasia of the cervix uteri and contraceptiona possible adverse effect of the pill. Lancet 1983;ii:930-4.

DHSS. On the state of the public health. London: DHSS, 1980:80.

Survey conducted by Taylor Nelson Medical for Schering Chemicals into contraceptive usage patterns, 1984

Bone M. Family planning services in England and Wales. London: HMSO, 1973. Lewton J. The changing pattern of male and female sterilisation: services, organisation, procedures.
Birth Control Trust, 1983 .

\section{Pharmacoepidemiology: a new discipline}

The safety of medicines is never far from public gaze, yet 16 years after the Medicines Act 1968 established the Committee on the Safety of Medicines leading articles in medical journals show that the medical profession is far from satisfied with current methods of assessing adverse reactions to drugs. ${ }^{12}$ The Centre for Medicines Research held a one day international meeting on the subject last autumn, which concluded that no single system, present or prospective, could be relied on as a sole method. ${ }^{3}$

That meeting led to another, intended to examine the issue in greater depth, which took the form of a four day residential seminar at Minster Lovell. Its main conclusion was that drug surveillance requires no less than the recognition and fostering of a new discipline-pharmacoepidemiology. This discipline already exists in embryo in Britain and the United States but is rarely acknowledged as such. The name seems lengthy but it is needed for a proper definition of the two essential disciplines-pharmacological, defining both beneficial as well as adverse drug effects, and epidemiological, studying the response of the population to these effects.
What claims make this subject a discipline or even a clinical specialty? The Minster Lovell seminar clearly identified these. Firstly, skill is needed to understand and allow for the inherent limitations of the information available, owing to problems of bias and imprecision of data (especially in comparison with laboratory standards) and to recognise that some questions cannot be answered. Secondly, organisation and costing have to be related to the likely benefit. Thirdly, the range of techniques available for collecting formal data must be understood-such as prospective and retrospective cohorts of patients, ${ }^{45}$ case-control studies, ${ }^{6}$ and linked data from hospitals ${ }^{7}$ or general practice ${ }^{8}$ or a combination of both in a group health scheme. ${ }^{9}$ Professional attention is also needed for the proper use of informal data from voluntary reporting. Fourthly, both the pharmacological action of drugs and the pathogenesis of disease need to be understood if these data are to be analysed and interpreted. For example, over 100 disease entities occur both naturally and as a response to drugs and only a few are unique to drugs. Lastly, specialised epidemiological concepts and methods need to be understood - the exposure of population groups to drugs, induction theory, the multicausal nature of events, hypothesis formation, statistical testing, and the use of models.

If progress is to be made we must create full time professionals with this specialised knowledge to work in academic units, in the pharmaceutical industry, and in government drug regulatory agencies to meet the public health, social, and even political problems that stem from adverse reactions to drugs. Such specialists in pharmacoepidemiology, though of great importance, may well be few since the problem of adverse drug effects appears to be modest in relation to the benefits that medicines confer. ${ }^{10} 11$

The Minster Lovell seminar concluded with a discussion of the experience of the pharmaceutical industry in the United States and in Britain with the use of newer techniques in developing drugs. Several banks of computerised data are now available in North America, some of which may be used to link drug prescriptions with clinical information, offering a powerful tool for studying drug effects. Britain does not have this facility for the whole country, and this gap in resources among countries is likely to widen unless specific action is taken to close it. The seminar recognised that worthy efforts have been made in Britain with cohorts and case-control studies, but modern techniques of collecting and processing could allow future studies to be done more quickly with more accuracy and at less cost.

Recently the main regulatory decisions in Britain have been made solely on voluntary "yellow card" data. If the proper resources had been available other methods would have provided a chance to test quantitatively, quickly, and cheaply whether the new effects reported were indeed drug induced. Ideally drug monitoring should be conducted in three distinct stages: after licensing (on the basis of information from laboratory and clinical studies) the routine first phase would consist of continued clinical trials; spontaneous reports (yellow cards and others); and study of cohorts of patients registered by gaining access to information on prescription forms. ${ }^{45}$ If an alert on safety grounds was raised by any of these methods and the relation between drug and event was not plain immediately and unequivocally, a second phase could be introduced with further spontaneous reports of the suspected reaction, new cohorts or retest of previous ones, case-control studies, and review of death and disease registers. In each case the choice of the appropriate technique(s) would depend on circumstances. If the alert appeared to be confirmed yet a third phase could be entered, 
in which the incidence of the effect would be measured so that a careful benefit-risk assessment could be formed, taking account of information on other similar drugs. Resources giving rapid and accurate assessments on these lines would allow better decisions about products by both companies and regulatory authorities.

Perhaps the most important conclusion of the Minster Lovell seminar was that an automated national data base linking prescriptions filled with hospital diagnoses in Britain would be extremely valuable in allowing the rapid study of drug effects and providing quantitative data. This would lessen public anxiety and restore much needed confidence. The Prescription Pricing Authority and the National Health Service hospital system provide an opportunity for such a system of drug surveillance based on computerised linkage of prescriptions with hospital diagnoses. A start has been made in the Dundee record linkage study ${ }^{3}$ and the cimetidine postmarketing surveillance study. ${ }^{4}$ Confidentiality should not be allowed to be an insuperable problem, and the Minster Lovell seminar also concluded that technical and organisational barriers could be overcome-provided that enough individuals were encouraged to make a commitment to research in pharmacoepidemiology.

Consultant Physician

D H LAwSON

Royal Infirmary

Glasgow G4 OSF

1 Turner P. Long-term assessment of drug safety and efficacy. $\mathcal{F}$ R Soc Med 1984;77:93-4

2 Rawlins MD. Postmarketing surveillance of adverse reactions to drugs. Br Med f 1984;288:87980

Walker SR, Goldberg A, eds. Montoring for adverse drug reactions. Lancaster: MTP Press, 1984 4 Colin-Jones DG, Langman MJS, Lawson DH, Vessey MP. Postmarketing surveillance of the safety of cimetidine: 12 month mortality report. Br Med J 1983;286:1713-6.

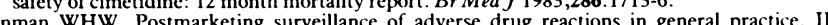
Prescription monitoring at the University of Southampton. Br Med I 1981;282:1216-7.

Mann JI, Vessey MP, Thorogood M, Doll R. Myocardial infarction in young women with special reference to oral contraceptive practice. Br Med 7 1975;ii:241-4

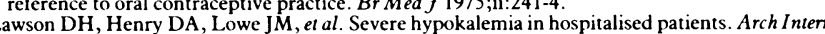
Med 1979;139:978-80.

Skegg DCG, Loll R. Frequency of eye complaints and rashes amongst patients receiving practolo and propranolol. Lancel 1977; ii:475-8.

ick H, Watkins RN, Hunter JR, et al. Replacement estrogens and endometrial cancer. N Engl 7 Med 1979;300:218-22.

10 Jick H. Drugs-remarkably non-toxic. N Engl f Med 1974;291:824-8

WHW. Risks in medical intervention: balancing therapeutic risks and benefits. Oxford: University of Oxford, 1984. (Wolfson lecture, 1984.)

\section{Uncommon haemophilus infections}

Haemophilus influenzae is a familiar cause of meningitis and epiglottitis-two diseases which present dramatically and, untreated, threaten survival. Otitis media, sinusitis, and infective exacerbations of chronic bronchitis are also frequently caused by $H$ influenzae, though non-encapsulated strains predominate in chest infections. What is less well known is the range of other infections that may be caused by this organism. A recent review from Finland has shown that in $17 \%$ of patients with bacteraemia with $H$ influenzae the infection was in sites other than the meninges and epiglottis. ${ }^{1}$ The joints, bones, eyes, skin, parotids, ${ }^{2}$ the genitourinary tract, ${ }^{3}$ and the heart valves need to be added to the list. Invasion of the blood stream is generally secondary to infection in these sites, but it may occur in isolation.

Infections caused by encapsulated $H$ influenzae occur predominantly in early childhood. Septic arthritis, cellulitis, and bacteraemia tend to affect children under 2 years of age, while epiglottitis and pneumonia are more frequent in those between 2 and 5 . The nadir of passively acquired maternal antibody plays a part in this vulnerability to infection but is by no means the whole explanation. Indeed, the picture is further confused by the occurrence of arthritis, ${ }^{4}$ meningitis, ${ }^{5}$ septicaemia, ${ }^{6}$ and pneumonia ${ }^{78}$ due to $H$ influenzae in older children and in adults.

Septic arthritis usually affects the larger joints such as the knee, ankle, and hip, and occasionally those of the arm. ${ }^{9}$ The features are those of an acute pyoarthrosis with pain (which may be referred), limitation of movement, swelling, and variable erythema. Fever is usual, sweating common, and rigors an occasional accompaniment. The younger child may be unwell in a non-specific way and fractious. Reluctance to use a limb is an important clue. An inferted purulent exudate at arthrocentesis confirms the diagnosis. Osteomyelitis of the adjacent bone is rare, as is spread to other joints. Rest, analgesics, antibiotics, and judicious arthrocenteses summarise the management.

$H$ influenzae may cause a cellulitis that usually affects the cheek, occasionally the periorbital area, and may follow a coryzal prodrome. ${ }^{10} 11$ The area is raised, warm, and tender and may be purplish rather than red. Fever, sweating, and generalised toxicity are common. The anatomical distribution and purplish colour provide a clue to the nature of this infection, which must be differentiated from streptococcal pyoderma, trauma, and the slapped cheek syndrome, otherwise known as erythema infectiosum or fifth disease. ${ }^{12} 13$

The true incidence of pneumonia caused by encapsulated strains of $H$ influenzae is unknown, since in the absence of bacteraemia this infection can rarely be diagnosed with confidence by sputum culture. None the less, $H$ influenzae is well described as an occasional cause of pneumonia in adults, ${ }^{7814}$ and accounted for $3 \%$ of infections in a recent study of adult pneumonia in Britain, while $2 \%$ of the bacteraemic infections in the Finnish study complicated pneumonia. ${ }^{1}$ Pneumonia in early childhood may be caused more frequently than has been thought by $H$ influenzae according to recent antigen detection studies. ${ }^{16}$

The heart is rarely the target for $H$ influenzae infection; pyogenic pericarditis may occur in isolation or complicate respiratory infection, meningitis, or epiglottitis, ${ }^{17}$ and endocarditis occurs uncommonly and has no distinguishing features. ${ }^{18}$ Other less common infections include conjunctivitis, enophthalmitis, subcutaneous abscess, peritonitis, glossitis, thyroiditis, laryngotracheobronchitis without epiglottitis, and biliary tract infections.

Laboratory diagnosis of $H$ influenzae infections is not difficult, although its pleomorphic Gram negative staining characteristics may make it difficult to visualise against a background of pus. Type b capsular polysaccharide may be identified with potent antiserum. Rapid methods for detecting this capsular polysaccharide are gaining in popularity and may have a bearing on the early management of these infections. Various body fluids such as serum, cerebrospinal fluid, and urine have been tested for type b capsular antigen by counterimmunoelectrophoresis, coagglutination, and latex agglutination. ${ }^{16} 19$

Treatment of $H$ influenzae infections has been made more difficult with the increasing resistance to ampicillin. A recent survey has shown that some $6 \cdot 2 \%$ of isolates in Britain were resistant, though there was considerable geographical variation. ${ }^{20}$ Chloramphenicol has well known undesirable properties, and resistance among $H$ influenzae, though still uncommon, is well recognised. ${ }^{22}$ Drugs such as the injectable cephalosporins seem iikely to be used more frequently. Among those that are highly active against 